\title{
How to manage ischaemic mitral regurgitation
}

Patrizio Lancellotti, Thomas Marwick and Luc A Pierard

Heart 2008;94;1497-1502

doi:10.1136/hrt.2007.134833

Updated information and services can be found at:

http://heart.bmj.com/cgi/content/full/94/11/1497

These include:

References This article cites 34 articles, 25 of which can be accessed free at: http://heart.bmj.com/cgi/content/full/94/11/1497\#BIBL

1 online articles that cite this article can be accessed at: http://heart.bmj.com/cgi/content/full/94/11/1497\#otherarticles

Rapid responses You can respond to this article at: http://heart.bmj.com/cgi/eletter-submit/94/11/1497

Email alerting Receive free email alerts when new articles cite this article - sign up in the box at service the top right corner of the article

Topic collections Articles on similar topics can be found in the following collections

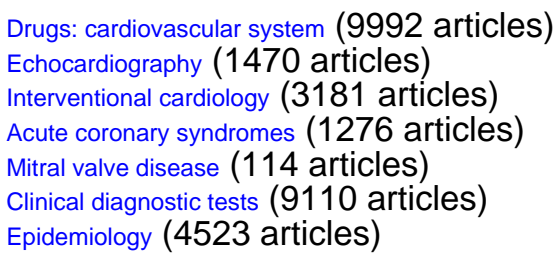

Notes

To order reprints of this article go to: http://journals.bmj.com/cgi/reprintform

To subscribe to Heart go to:

http:/journals.bmj.com/subscriptions/ 


\title{
How to manage ischaemic mitral regurgitation
}

\author{
Patrizio Lancellotti, ${ }^{1}$ Thomas Marwick, ${ }^{2}$ Luc A Pierard ${ }^{3}$
}

\begin{abstract}
${ }^{1}$ Responsable de I'Unité de Soins Intensifs Cardiologiques, CHU Sart Tilman, Liege, Belgique; ${ }^{2}$ Department of Cardiology, Princess Alexandra Hospital and University of Queensland School of Medecine, Brisbane, Australia; ${ }^{3}$ Faculté de Médecine, Université de Liège, Chef de Service, Service de Cardiologie, CHU Sart Tilman, Liege, Belgique
\end{abstract}

Correspondence to: Professor Patrizio Lancellotti، Department of Cardiology, University Hospital of Liège, B - 4000 Liege, Belgium; plancellotti@chu.ulg.ac.be
Ischaemic heart disease is becoming an increasingly frequent cause of ischaemic mitral regurgitation (IMR). Three different clinical entities of IMR, which deeply affect the clinical decision making, are distinguishable: the acute IMR complicating an acute myocardial infarction, the true IMR secondary to a transient ischaemic phenomenon, and the chronic functional IMR (FIMR). The incidence of the two first entities is low; the third is much more frequent.

\section{ACUTE ISCHAEMIC MITRAL REGURGITATION COMPLICATING THE ACUTE PHASE OF MYOCARDIAL INFARCTION}

IMR can occur acutely in patients sustaining an acute myocardial infarction. The rupture of a papillary muscle-most frequently a head of a posteromedial papillary muscle-is a dramatic mechanical complication of acute myocardial infarction, leading to a very high mortality rate in the absence of immediate surgical intervention. Surgery, most often valve replacement, is warranted after stabilisation of the haemodynamic status using an intra-aortic balloon pump and vasodilators. ${ }^{1}$ In the absence of such a rupture, the presence of IMR in the acute myocardial infarction phase portends a grave prognosis. Its incidence and clinical importance are largely underestimated, partly because physical examination is rather insensitive. Prompt in-hospital revascularisation can prevent or reverse acute IMR. ${ }^{2}$ When limited to the inferior wall, early revascularisation may reduce localised left ventricular (LV) remodelling and IMR. ${ }^{3}$ Early revascularisation also increases survival in patients with acute IMR presenting with shock. ${ }^{4}$

\section{TRUE ISCHAEMIC MITRAL REGURGITATION}

This second form truly defines IMR because it is secondary to an active ischaemic episode. It is clinically most often revealed by a "whistling angina" or a "flash pulmonary oedema". It is linked to the presence of a significant stenosis of the right or left circumflex coronary artery. In this situation, the primary treatment is to prevent the episodes of active myocardial ischaemia by a revascularisation procedure.

\section{CHRONIC FUNCTIONAL ISCHAEMIC MITRAL REGURGITATION}

Chronic FIMR, the most common cause of IMR, broadly denotes abnormal function of normal leaflets in the context of impaired ventricular function resulting from ischaemic heart disease. It results from an imbalance between tethering forces (annular dilatation, LV dilatation, papillary muscles displacement, LV sphericity) and closing forces (reduction of LV contractility, global LV dyssynchrony, papillary muscle dyssynchrony, altered mitral systolic annular contraction). ${ }^{5}$ Chronic FIMR results, in $95 \%$ of cases, from a type IIIb dysfunction (restricted leaflet motion). It is important to note that in the remaining $5 \%$, an ischaemic elongation of the papillary muscle may cause prolapse in the corresponding area (type II dysfunction). When present, FIMR may exhibit a broad range of severity and, when severe (effective regurgitant orifice $(\mathrm{ERO}) \geqslant 20 \mathrm{~mm}^{2}$ ), conveys a poor outcome. FIMR is characteristically dynamic during exercise. It has been shown that an increase $\left(\Delta \mathrm{ERO} \geqslant 13 \mathrm{~mm}^{2}\right)$ in FIMR during exercise identifies a subgroup of patients at higher risk of cardiac events. $^{67}$

\section{MANAGEMENT OF FUNCTIONAL ISCHAEMIC MITRAL REGURGITATION}

When treating FIMR, it is important to integrate several clinical parameters-symptoms of heart failure, episodes of worsening heart failure, comorbidities - and echocardiographic elements - mitral regurgitation severity at rest and its dynamic nature at exercise, the degree of mitral valve apparatus deformation, the origin and direction of regurgitant jets, the importance of LV remodelling, the presence of LV dyssynchrony, the presence and extent of viable myocardium at jeopardy. Different clinical scenarios might thus largely modulate the clinical decision making. Practically, instead of medical treatment, unbalanced tethering can be addressed by mitral valve surgery or by LV reshaping, whereas coronary revascularisation or cardiac resynchronisation therapy can potentially reduce tethering as well as increase mitral valve closing forces (fig 1).

\section{Medical treatment}

The scope of medical treatment, which remains the first step in the management of these patients, is to prevent myocardial ischaemia, reduce the severity of FIMR, and revert or delay the LV remodelling process. However, presently no pharmacological approach has been shown to improve the patient clinical outcome. Usual medical treatment of heart failure, including angiotensin converting enzyme 
Clinical: HF symptoms, decompensated HF, medical treatment (ACE inhibitor, $\beta$-blocker, spironolactone), comorbidities

Echo: $E R O \geqslant 20 \mathrm{~mm}^{2}$, dynamic FIMR ( $\Delta \mathrm{ERO}>13 \mathrm{~mm}^{2}$ at exercise) LV remodelling and sphericity, mitral valve deformation viability \pm ischaemia, LV dyssynchrony
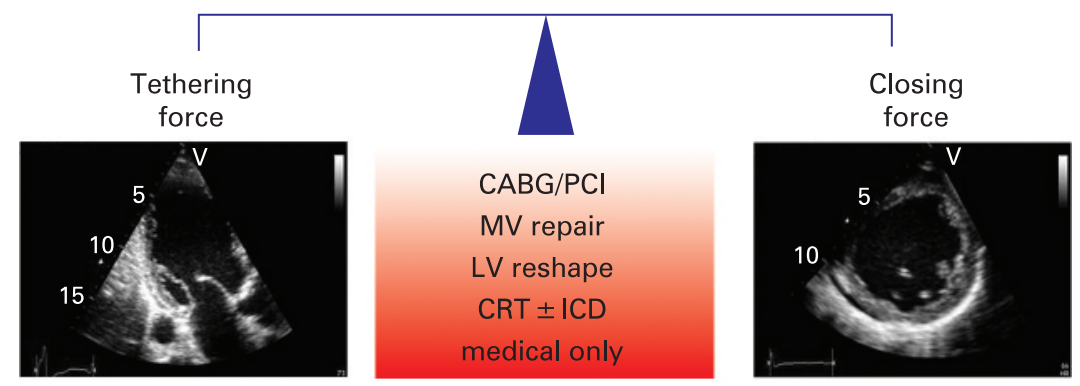

Figure 1 Global management of functional ischaemic mitral regurgitation. $A C E$, angiotensin converting enzyme; CABG, coronary artery bypass grafting; CRT, cardiac resynchronisation therapy; ERO, effective regurgitant orifice; FIMR, functional ischaemic mitral regurgitation; HF, heart failure; ICD, implantable cardioverter-defibrillator; LV, left ventricle; MV, mitral valve; $\mathrm{PCl}$, percutaneous coronary intervention.

(ACE) inhibitors, $\beta$-blockers and spironolactone, should be prescribed according to the official recommendations. All these drugs gradually lead to a fundamental reshaping of the LV. Diuretics are administered according to the signs of water retention. In cases of acute dyspnoea, sublingual nitrates seem to be beneficial. In practice, FIMR appears to remain common despite the use of these drugs. ${ }^{8}$

\section{Cardiac resynchronisation therapy}

Currently, when a revascularisation procedure is not indicated or insufficient to improve the clinical status, the use of biventricular pacing is justified (European Society of Cardiology (ESC) guidelines class I level of evidence A) in heart failure patients with significant ventricular conduction delay (ORS $>120 \mathrm{~ms}$ ) who remain symptomatic despite optimal drug treatment.9 The effects of cardiac resynchronisation therapy (CRT) on FIMR can be observed acutely or only late after implantation. This treatment is associated with an immediate reduction in the quantified degree of FIMR (expected reduction of $35 \%$ ), which is directly

Table 1 Indications for surgery in ischaemic mitral regurgitation (IMR)

\begin{tabular}{|c|c|}
\hline Clinical situation & Mitral valve surgery (ESC guidelines 2007) \\
\hline \multicolumn{2}{|l|}{ Patients with severe IMR (ER0 $\geqslant 20 \mathrm{~mm}^{2}$ ) } \\
\hline Ruptured papillary muscle due to acute MI & Immediate \\
\hline LV ejection fraction $\geqslant 30 \%$ undergoing CABG & Recommended (IC) \\
\hline LV ejection fraction $<30 \%$ and option for CABG & Reasonable if symptomatic (IlaC) \\
\hline LV ejection fraction $\geqslant 30 \%$, no option for CABG & $\begin{array}{l}\text { To consider if symptomatic and low morbidity } \\
\text { (llbC) }\end{array}$ \\
\hline \multicolumn{2}{|l|}{ Patients with moderate IMR (ERO $<20 \mathrm{~mm}^{2}$ ) } \\
\hline Undergoing CABG & Reasonable if repair is feasible (llaC) \\
\hline No option for $\mathrm{CABG}$ or undergoing $\mathrm{PCl}$ & Uncertain ("a priori not") \\
\hline \multicolumn{2}{|l|}{ Patients with trace IMR } \\
\hline Undergoing CABG & Not recommended \\
\hline
\end{tabular}

CABG, coronary bypass grafting; ERO, effective regurgitant orifice; ESC, European Society of Cardiology; LV, left ventricular; $\mathrm{MI}$, myocardial infarction; $\mathrm{PCl}$, percutaneous coronary intervention. correlated to the improvement in LV systolic performance (greater mitral closing force)increase in $\mathrm{LV} \mathrm{dP} / \mathrm{dt}$ - and the reduction in the interpapillary muscle activation time delay (less tension on mitral leaflets). ${ }^{10}{ }^{11}$ Reduction in mitral regurgitation severity also results from a more effective mitral annulus contraction and a significant decrease in systolic tenting area-the systolic displacement of the body of the mitral leaflets into the LV cavity. Thus, in the acute stage, CRT corrects the imbalance between forces acting on the mitral valve and enhances the sphincteric action of the annulus. It should be noted that these changes are often insufficient to remove regurgitation. Persistent residual FIMR is determined by the extent of residual deformation of the mitral valve. ${ }^{12}$

Besides these acute effects, an additional 10-20\% reduction in FIMR, that parallels the decrease in LV volumes and the increase in $\mathrm{LV} \mathrm{dP} / \mathrm{dt}$, occurs some months after CRT. Although this improvement is sustained at 1 year follow-up, it could be attenuated in some patients secondary to the progression of the ischaemic disease. ${ }^{13}$ The benefit of CRT appears to be dependent on continued pacing because withholding pacing results in an immediate loss of efficacy and recurrence of FIMR. Indeed, the transient interruption of CRT after long term results in acute deterioration of FIMR as a consequence of a pronounced decline in $\mathrm{LV} \mathrm{dP} / \mathrm{dt}$ and the reappearance of significant papillary muscle dyssynchrony. ${ }^{14}$ This supports the notion of maintaining CRT indefinitely. The effect of CRT on dynamic FIMR is also progressive and appears in the chronic stage of stimulation. Indeed, CRT reduces not only FIMR at rest but also its dynamic component during exercise. The expected attenuation of exercise induced FIMR is about $30 \% .{ }^{12}{ }^{15}$ However, CRT reduces the amount of increase in FIMR but does not avoid the development of at least some dynamic component in most patients.

The response to CRT might be modulated by the severity of FIMR and the extent of LV remodelling before implantation. Severe FIMR (ERO $\geqslant 20 \mathrm{~mm}^{2}$ ) in the setting of an enlarged LV is associated with lack of reverse remodelling during follow-up. Patients with more severe disease are thus less likely to respond to CRT. Moreover, the degree to which the reduction in FIMR plays a role in reverse LV remodelling remains currently unclear. CRT reduces but does not abolish FMR in most patients. The persistence of a critical level of regurgitation might thus be able to perpetuate the LV remodelling process. Data concerning long term effects are limited. Importantly, this mode of treatment is restricted to patients who fulfil the classical criteria for CRT.

\section{Surgical treatment}

The indication for surgical correction of FIMR is a source of debate. In most cases, coronary bypass grafting alone is not sufficient to remove regurgitation or even to reduce its severity. ${ }^{16}$ However, 
Table 2 Predictors of outcome after mitral valve surgery

\begin{tabular}{llll}
\hline History & Condition & Echocardiography & Surgery \\
\hline Older age & & LV ejection fraction $<30 \%$ & \\
Diabetes mellitus & High Euroscore & Extensive scar + no viability & Left main stenosis \\
Cardiac heart failure & Emergent operation & Severe LV remodelling & Three vessel disease \\
Increasing NYHA class & Cardiogenic shock & Severe MV deformation & MV replacement \\
Renal failure & & Complex regurgitant jet & Residual IMR \\
Associated diseases & & Restrictive diastolic filling & \\
\hline
\end{tabular}

IMR, ischaemic mitral regurgitation; LV, left ventricular; MV, mitral valve; NYHA, New York Heart Association.

not treating FIMR exposes the patient to a higher risk of hospitalisation for heart failure. ${ }^{17}$ The possible survival impact of mitral valve surgery is poorly known; mortality at 5 years remains high. ${ }^{18}$ Results from the literature unfortunately reflect their retrospective nature, the inevitable patient selection bias, and unresolved issues such as the best surgical technique to use. So far, the added value of valve repair compared to bypass surgery alone is thus uncertain. Table 1 lists the indications for surgery, and table 2 the predictors of outcome following surgery.

Practically, the decision to intervene in the mitral valve will depend on the severity of FIMR and whether or not bypass grafting will be scheduled. Unanimously, severe FIMR (ERO $\geqslant 20 \mathrm{~mm}^{2}$ ) should be corrected at the time of bypass surgery (ESC level IC), ${ }^{8}$ while trivial FIMR (ERO $<10 \mathrm{~mm}^{2}$ ) can be neglected. Remodelling annuloplasty using a prosthetic undersized ring (one or two sizes) is the technique of choice in type

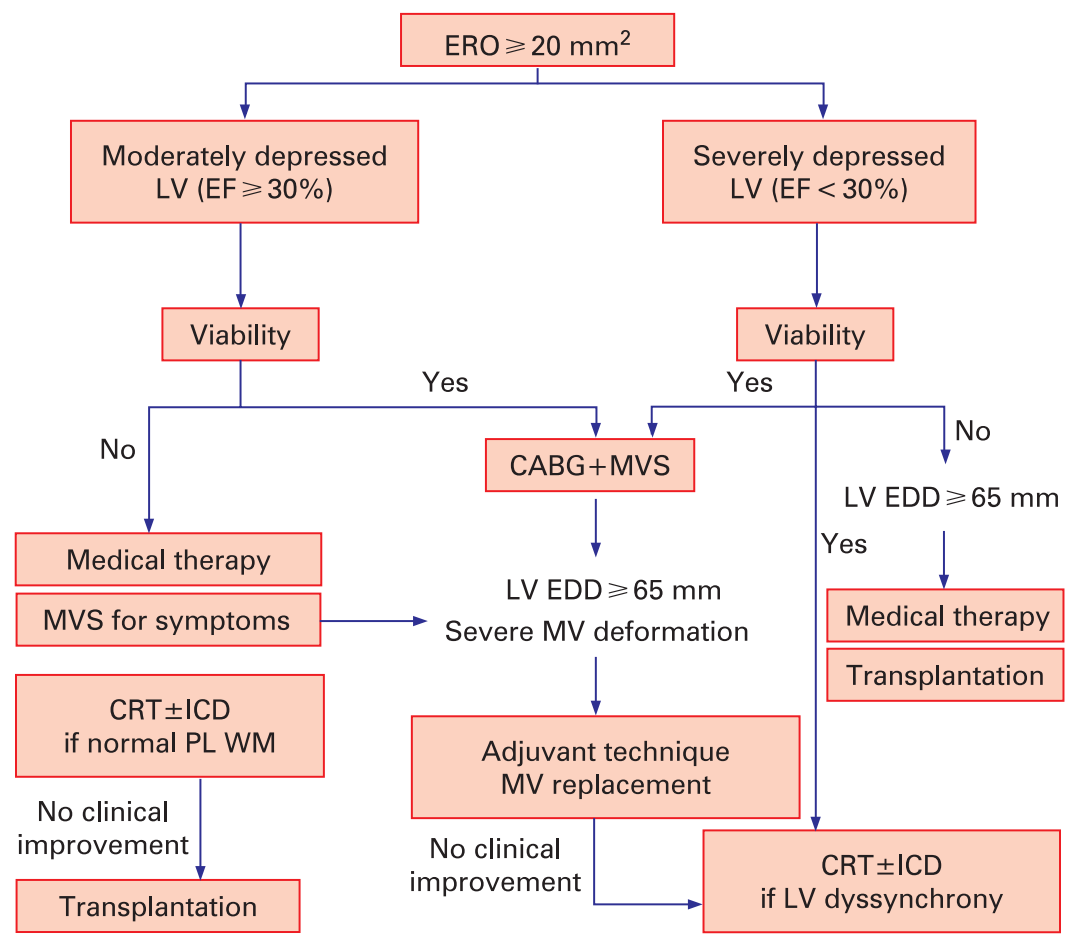

Figure 2 Management of patients with severe functional ischaemic mitral regurgitation. CABG, coronary artery bypass grafting; CRT, cardiac resynchronisation therapy; $E D D$, end-diastolic diameter; $E F$, ejection fraction; $E R O$, effective regurgitant orifice; ICD, implantable cardioverter-defibrillator; LV, left ventricle; MV, mitral valve; MVS, mitral valve surgery; PL WM, posterolateral wall motion.
IIIb dysfunction. ${ }^{19}$ If the repair is unfeasible (limited cases) or there is type II dysfunction in which stringent annuloplasty will be often inefficient, it is reasonable to advocate mitral valve replacement (bioprosthesis) with preservation of the subvalvular apparatus. In symptomatic patients with severe FIMR and no option for revascularisation, mitral valve repair may be considered (ESC level IIbC). However, when the LV function is severely impaired, surgery is more likely to be considered if myocardial viability is present, if comorbidity is low, if FIMR increases dynamically during exercise, if the LV is not severely remodelled, and if the revascularisation can be almost complete. In the other cases, cardiac transplantation (ESC level IC) remains the preferable approach since it is associated with a better 5 year outcome (fig 2).

In patients with moderate FIMR (ERO $<20 \mathrm{~mm}^{2}$ ), the ischaemic symptoms usually dictate the treatment strategy. For example, mildly symptomatic patients with no residual myocardial ischaemia and mild to moderate FIMR will be usually treated medically, whereas symptomatic patients with active or inducible ischaemia will be oriented towards a revascularisation procedure. When percutaneous coronary revascularisation is performed, mitral valve surgery is rarely proposed. In this situation, the patient should be carefully followed up to detect any FIMR progression and ongoing LV remodelling process. Conversely, when bypass grafting is planned, the decision to repair the mitral valve remains a matter of controversy. In such cases, if the mitral valve is repairable, combined surgery is reasonable (ESC level IIaC). However, the additional risk of mitral valve surgery is not negligible, particularly in patients with comorbidities and poor LV function. Very ill patients should be treated conservatively with revascularisation alone. ${ }^{17}$ In the future percutaneous annuloplasty may possibly be proposed for these patients. ${ }^{20}$ Low risk patients can be further stratified with exercise echocardiography. ${ }^{16}$ Although this technique is of growing interest, its value to predict the results of surgery still remains to be evaluated. Patients with a dynamic increase in FIMR $\left(\Delta E R O \geqslant 13 \mathrm{~mm}^{2}\right)$ during exercise might be submitted for combined surgery, while patients with either no significant changes or exercise induced reduction in FIMR as a result of recruitable function in the inferobasal wall might be referred for bypass grafting alone (fig 3).

Several groups have used transoesophageal echocardiography at the beginning of the intervention to make this decision. However, because of changes in loading conditions, general anaesthesia leads almost systematically to underestimation of FIMR severity (reduction of moderate FIMR to trace amounts). ${ }^{21}$ Accordingly, to minimise the effects of anaesthetic induction on FIMR some groups perform a preload and afterload challenge test. A rapid fluid filling is performed until the mean capillary wedge pressure reaches $15-18 \mathrm{~mm} \mathrm{Hg}$. If the regurgitation does not 


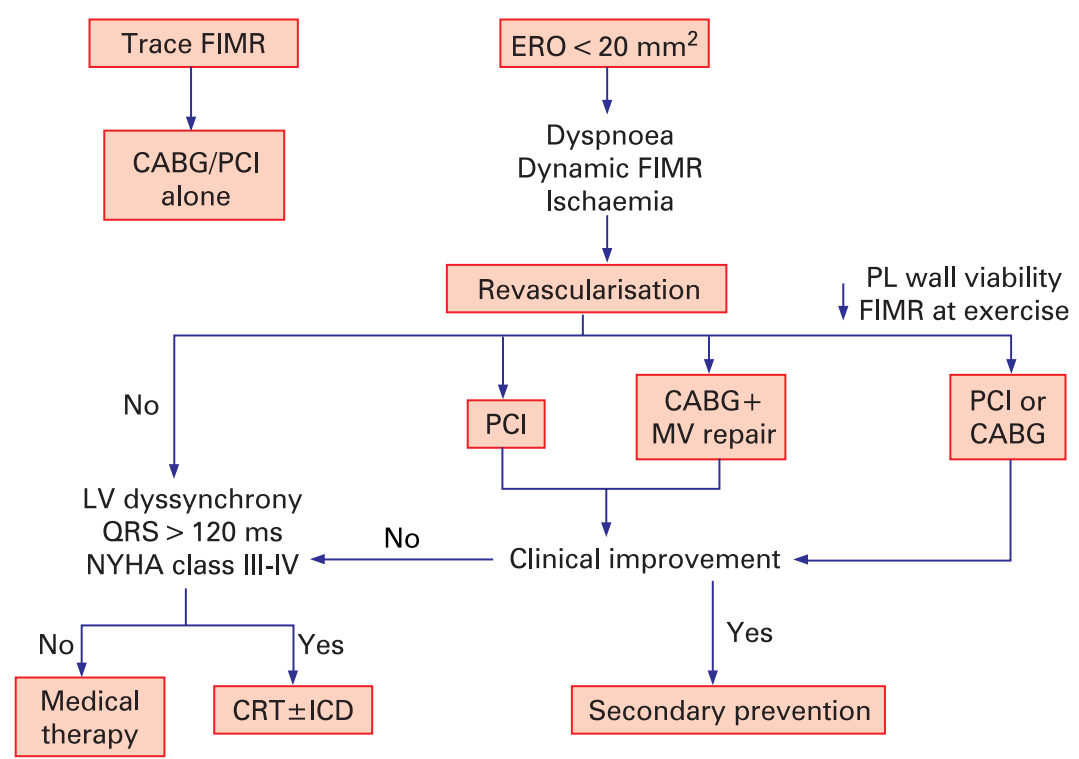

Figure 3 Management of patients with moderate functional ischaemic mitral regurgitation. $C A B G$, coronary artery bypass grafting; $C R T$, cardiac resynchronisation therapy; ERO, effective regurgitant orifice; FIMR, functional ischaemic mitral regurgitation; ICD, implantable cardioverter-defibrillator; $L V$, left ventricle; MV, mitral valve; NYHA, New York Heart Association; PCl, percutaneous coronary intervention; $\mathrm{PL}$, posterolateral.

increase, phenylephrine is administered until the mean arterial blood pressure reaches $100 \mathrm{~mm} \mathrm{Hg} .{ }^{18} 21$ This approach provides a rough assessment of the dynamic behaviour of FIMR. Indeed, the level of increase in the FIMR associated with the need for repair is not yet validated.
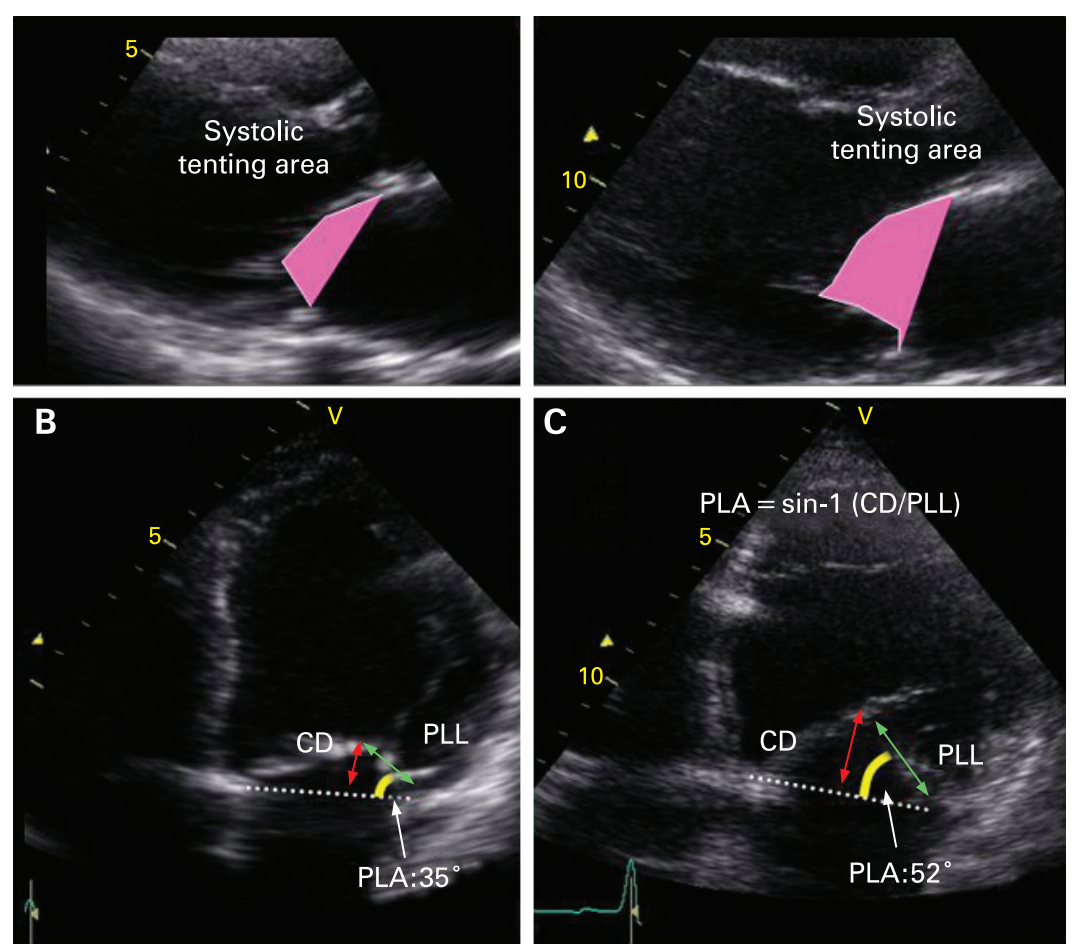

Figure 4 Echocardiographic assessment of mitral valve deformation. CD, coaptation distance (red arrow); PLA, posterior leaflet angle; PLL, posterior leaflet length (green arrow).
Table 3 Preoperative predictors of mitral valve repair failure (transthoracic echocardiography)

\begin{tabular}{ll}
\hline Parameters & Threshold \\
\hline Coaptation distance & $\geqslant 1 \mathrm{~cm}$ \\
Systolic tenting area & $>2.5-3 \mathrm{~cm}^{2}$ \\
Posterolateral angle & $\geqslant 45^{\circ}$ \\
Lateral wall motion abnormality & Scar \\
Central regurgitant jet & - \\
Complex regurgitant jets & Multiple \\
Left ventricular end-diastolic diameter & $\geqslant 65 \mathrm{~mm}$ \\
Left ventricular end-systolic diameter & $\geqslant 51 \mathrm{~mm}$ \\
\hline
\end{tabular}

Practically, it is probably best to identify potential candidates for combined surgery before intervention.

Residual or recurrent FIMR after mitral valve surgery It has become increasingly clear that a ventricular problem cannot be resolved by a single annular procedure - that does not directly address tethering by the remodelled LV-in every case. ${ }^{5}$ Persistence or recurrence of FIMR is observed in approximately one third of patients, the risk being more significant during the first 6 months after surgery (table 3). ${ }^{516}$ The persistence of FIMR — which can perpetuate the LV remodelling process and adversely affect the patient's outcome-could be explained by the postoperative persistence and/or worsening of mitral leaflets tethering. ${ }^{5}$ In most instances, mitral ring annuloplasty reduces the anterior-posterior annular dimension and contributes to restoring a surface of coaptation. ${ }^{19}$ By doing so, it always induces, for geometrical reasons, a further tethering of the posterior leaflet, but also of the anterior leaflet, and frequently converts the valve into a monocusp with sometimes a restricted anterior leaflet motion as well. ${ }^{22}$ This pattern of coaptation of the edge of the anterior leaflet against the body of the even more tethered posterior leaflet usually accounts for a mild residual leak postoperatively. This is particularly more pronounced in patients with an asymmetrical mitral orifice (P2-P3 are more restricted) who received a symmetrical ring. If the remodelling process is not halted by the procedure, a further tethering of both the anterior and posterior leaflet is expected with a recurrence of FIMR. ${ }^{5}$

Several echocardiographic parameters can help to identify patients at risk of treatment failure (fig 4). Preoperatively (transoesophageal echocardiography), patients with a mitral diastolic annulus diameter $\geqslant 37 \mathrm{~mm}$, a systolic tenting area $\geqslant 1.6 \mathrm{~cm}^{2}$ and an FIMR grade $>3.5$ have a $50 \%$ probability of failure during follow-up. ${ }^{23}$ Preoperatively (transthoracic echocardiography), a coaptation distance $>1 \mathrm{~cm}$ (distance between the leaflet coaptation point and the mitral annulus plane in the apical four chamber view), a systolic tenting area $>2.5 \mathrm{~cm}^{2}$ (the area enclosed between the annular plane and mitral leaflets in the parasternal long axis view), a posterior leaflet angle $\geqslant 45^{\circ}$ (indicating a high posterior leaflet 


\section{How to manage ischaemic mitral regurgitation: key points}

- Bypass grafting (CABG) alone does not correct functional ischaemic mitral regurgitation (FIMR) in most patients.

- The candidate for combined surgery (mitral valve replacement $+\mathrm{CABG}$ ) should be selected preoperatively.

- Undersized mitral annuloplasty is the technique of choice.

- Recurrent FIMR is frequent in patients with severe mitral valve deformation/ left ventricular remodelling.

- Survival advantage from repair rather than mitral valve replacement is evident in most patients.

- The precise role of subvalvular adjuncts needs to be studied.

- Prospective registries and randomised trials are necessary.

restriction), a central regurgitant jet (indicating a severe restriction of both leaflets in patients with severe FIMR), the presence of complex jets originating centrally and posteromedially, a restrictive diastolic filling profile (suggesting a more advanced LV disease process and associated with further negative LV remodelling after repair), and a severe LV enlargement (low likelihood of reverse LV remodelling after repair and poor late outcome) increase the risk of mitral valve repair failure. Taking these parameters altogether could reduce the frequency of postoperative FIMR. ${ }^{24}$ For example, using a two sizes stringent ring (ring 24-26), in conjunction with obtaining a coaptation distance $\leqslant 8 \mathrm{~mm}$ at the end of intervention in selected patients with a preoperative LV end diastolic volume $\leqslant 65 \mathrm{~mm}$, leads to a dramatic decrease in the incidence of recurrent FIMR as low as $2.3 \%{ }^{18}$ This could, however, increase the risk of inducing mitral stenosis. ${ }^{25}$ When the mitral valve is severely deformed and the LV significantly enlarged, it would be reasonable also to restore the geometry of the LV and/or to reduce directly the leaflets tethering by a complementary subvalvular or

\section{You can get CPD/CME credits for Education in Heart}

Education in Heart articles are accredited by both the UK Royal College of Physicians (London) and the European Board for Accreditation in Cardiologyyou need to answer the accompanying multiple choice questions (MCOs). To access the questions, click on BMJ Learning: Take this module on BMJ Learning from the content box at the top right and bottom left of the online article. For more information please go to: http://heart.bmj.com/misc/education. $\mathrm{dtl}$

RCP credits: Log your activity in your CPD diary online (http://www. rcplondon.ac.uk/members/CPDdiary/index.asp)—pass mark is $80 \%$.

- EBAC credits: Print out and retain the BMJ Learning certificate once you have completed the MCOs - pass mark is 60\%. EBAC/ EACCME Credits can now be converted to AMA PRA Category 1 CME Credits and are recognised by all National Accreditation Authorities in Europe (http://www.ebac-cme.org/ newsite/?hit $=$ men02).

Please note: The MCQs are hosted on BMJ Learning - the best available learning website for medical professionals from the BMJ Group. If prompted, subscribers must sign into Heart with their journal's username and password. All users must also complete a one-time registration on BMJ Learning and subsequently log in (with a BMJ Learning username and password) on every visit. ventricular approach. In some cases, mitral valve replacement is defendable.

\section{New treatment modalities}

This intrinsic limitation of simple reductive annuloplasty has been challenged by different techniques which aim at reducing the traction exerted from the LV on the mitral valve. ${ }^{26}$ Enlargement of the posterior leaflet, especially in the area of the posteromedial commissure, may enable the placement of a normal ring and restore a surface of coaptation by reducing the tethering. The surgical relocation of the posterior papillary muscle is another approach to correct the tethering at the ventricular level. ${ }^{27}$ Resection of secondary strut cords that account in some cases for bending of the anterior leaflet and reduced coaptation, although appealing, has not led to conclusive clinical results. ${ }^{28}$ The association of the edge-to-edge Alfieri procedure to an undersized annuloplasty seems to reduce the incidence of repair failure in patients with excessive tethering (coaptation distance $\geqslant 1 \mathrm{~cm}$ ). ${ }^{29}$ Asymmetrical tethering could be solved by using an asymmetrical ring annuloplasty. Severe LV remodelling could be challenged by an LV restoration using the modified Dor procedure or by applying the "Acorn" or the "Myosplint" system. ${ }^{30}$

More recently, various companies have launched studies on percutaneous approaches of mitral valve repair. The majority of devices (for example, MitraLife-Edwards, Edwards Monarc, Cardiac Dimensions, Viacor) take advantage of the proximity of the coronary sinus to the posterior mitral annulus to deliver devices (anchors that are placed in the anterior and posterior portions of the coronary sinus) that remodel the mitral annulus. ${ }^{31-33}$ The Edwards Monarc device consists of a pair of self expanding nitinol anchoring stents located at the ends of the device and joined to each other by a connecting element that undergoes delayed shortening over 3-4 weeks after placement. The Cardiac Dimensions Carillon device consists of two different sized, self expanding figure-of-eight nitinol loops located at either end of a fixed length stainless steel connector. The Viacor device is used to place one or more straightening rods within a catheter that is positioned in the coronary sinus via the subclavian vein. Although these rods do not plicate the coronary sinus per se, they do "bend" the sinus in a manner that pushes the posterior leaflet forward relative to the location of the commissures. Other devices such as the Mitralign perform septal lateral cinching by direct placement of several almost-stapled-like anchors around the mitral annulus to reduce the diameter of the orifice. The percutaneous septal sinus shortening approach (Ample Medical), the Quantun Cor, and the minimally invasive iCoapsys device are new percutaneous devices under evaluation. Finally, in an experimental model, it has been shown that cell transplantation in the infarcted area can also potentially reduce IMR through a decrease in localised LV wall 
deformation. ${ }^{34}$ So far, these techniques-which stress the importance of addressing the ventricular factors that cause FIMR - have only been applied in small clinical cohorts or are still limited to experimental models. Thus, definitive recommendations cannot be made at present.

Competing interests: In compliance with EBAC/EACCME guidelines, all authors participating in Education in Heart have disclosed potential conflicts of interest that might cause a bias in the article. The authors have no competing interests.

\section{REFERENCES}

1. Bursi F, Enriquez-Sarano M, Jacobsen SJ, et al. Mitral regurgitation after myocardial infarction: a review. Am J Med 2006;119:103-12.

- An excellent review on acute ischaemic mitral regurgitation.

2. Bursi F, Enriquez-Sarano M, Vuyisile $T$, et al. Heart failure and death after myocardial infarction in the community. The emerging role of mitral regurgitation. Circulation 2005;111:295-301.

3. Tenenbaum A, Leor J, Motro M, et al. Improved posterobasal segment function after thrombolysis is associated with decreased incidence of significant mitral regurgitation in a first inferior myocardial infarction. J Am Coll Cardiol 1995;25:1558.

4. Picard MH, Davidoff R, Sleeper LA, et al; SHOCK Trial. SHould we emergently revascularize Occluded Coronaries for cardiogenic shock. Echocardiographic predictors of survival and response to early revascularization in cardiogenic shock. Circulation 2003;107:279-84.

5. Levine RA, Schwammenthal E. Ischemic mitral regurgitation on the threshold of a solution: from paradoxes to unifying concepts. Circulation 2005;112:745-58.

- A nice review on mechanisms and treatments of acute and chronic ischaemic mitral regurgitation.

6. Lancellotti $\mathbf{P}$, Troisfontaines P, Toussaint A-C, et al. Prognostic importance of exercise-induced changes in mitral regurgitation in patients with chronic ischemic left ventricular dysfunction. Circulation 2003;108:1713-17.

- First clinical paper showing the prognostic impact of dynamic mitral regurgitation.

7. Piérard LA, Lancellotti $P$. The role of ischemic mitral regurgitation in the pathogenesis of acute pulmonary edema. N Engl J Med 2004;351:1627-34.

- First clinical paper showing that dynamic mitral regurgitation could represent a mechanism of acute pulmonary oedema.

8. Vahanian A, Baumgartner $\mathrm{H}$, Bax J, et al. Task Force on the Management of Valvular Hearth Disease of the European Society of Cardiology; ESC Committee for Practice Guidelines. Guidelines on the management of valvular heart disease: The Task Force on the Management of Valvular Heart Disease of the European Society of Cardiology. Eur Heart J 2007;28:230-68.

9. Authors/Task Force Members, Vardas PE, Auricchio A, et al. Guidelines for cardiac pacing and cardiac resynchronization therapy: the Task Force for Cardiac Pacing and Cardiac Resynchronization Therapy of the European Society of Cardiology. Developed in collaboration with the European Heart Rhythm Association. Eur Heart J 2007;28:2256-95.

10. Breithardt $\mathbf{O A}$, Sinha AM, Schwammenthal E, et al. Acute effects of cardiac resynchronization therapy on functional mitra regurgitation in advanced systolic heart failure. J Am Coll Cardiol 2003;41:765-70.

- Echocardiographic study examining the effects of CRT on mitral regurgitation.

11. Kanzaki H, Bazaz R, Schwartzman D, et al. A mechanism for immediate reduction in mitral regurgitation after cardiac resynchronization therapy. J Am Coll Cardiol 2004;44:1619-25.

12. Lancellotti $\mathbf{P}$, Mélon $P$, Sakalihasan N, et al. Effects of cardiac resynchronization therapy on functional mitral regurgitation in heart failure. Am J Cardiol 2004;94:1462-5.

13. Sutton MG, Plappert T, Hilpisch KE, et al. Sustained reverse left ventricular structural remodeling with cardiac resynchronization at one year is a function of etiology: quantitative Doppler echocardiographic evidence from the Multicenter InSync Randomized Clinical Evaluation (MIRACLE). Circulation 2006;113:266-72.

14. Ypenburg C, Lancellotti P, Tops LF, et al. Acute effects of initiation and withdrawal of cardiac resynchronization therapy on papillary muscle dyssynchrony and mitral regurgitation. J Am Coll Cardiol 2007; 50:2071-7.

- Echocardiographic study examining the effects of CRT on papillary muscle dyssynchrony and mitral regurgitation.

15. Madaric J, Vanderheyden M, Van Laethem C, et al. Early and late effects of cardiac resynchronization therapy on exercise-induced mitral regurgitation: relationship with left ventricular dyssynchrony, remodelling and cardiopulmonary performance. Eur Heart J 2007;28:2134-41.

- Study showing that the effects of CRT on dynamic mitral regurgitation is gradual.

16. Lancellotti $\mathbf{P}$, Donal E, Cosyns B, et al. Effects of surgery on ischaemic mitral regurgitation: a prospective multicenter registry (SIMRAM registry). Eur J Echocardiogr 2008;9:26-30.

17. Kang DH, Kim MJ, Kang SJ, et al. Mitral valve repair versus revascularization alone in the treatment of ischemic mitral regurgitation. Circulation 2006;114:1499-503.

18. Braun J, van de Veire NR, Klautz RJ, et al. Restrictive mitral annuloplasty cures ischemic mitral regurgitation and heart failure. Ann Thorac Surg 2008;85:430-6.

- Important clinical study showing that restrictive annuloplasty could be sufficient to treat ischaemic mitral regurgitation in highly selected patients.

19. Bolling SF, Pagani FD, Deeb GM, et al. Intermediate-term outcome of mitral reconstruction in cardiomyopathy. J Thorac Cardiovasc Surg 1998;115:381-6.

20. Webb JG, Harnek J, Munt Bl, et al. Percutaneous transvenous mitral annuloplasty. Initial human experience with device implantation in the coronary sinus. Circulation 2006;113:851-5.

- Human experience of percutaneous transvenous mitral valve annuloplasty.

21. Gisbert A, Souliere V, Denault AY, et al. Dynamic quantitative echocardiographic evaluation of mitral regurgitation in the operating department. J Am Soc Echocardiogr 2006;19:140-6.

22. Zhu F, Otsuji Y, Yotsumoto G, et al. Mechanism of persistent ischemic mitral regurgitation after annuloplasty: importance of augmented posterior mitral leaflet tethering. Circulation 2005; 112:I396-401.

23. Kongsaerepong V, Shiota M, Gillinov AM, et al. Echocardiographic predictors of successful versus unsuccessful mitral valve repair in ischemic mitral regurgitation. Am J Cardiol 2006;98:504-8

- Echocardiographic study examining the predictors of mitral valve repair failure.

24. Magne J, Pibarot Ph, Dagenais F, et al. Preoperative posterior leaflet angle accurately predicts outcome after restrictive mitral valve annuloplasty for ischemic mitral regurgitation. Circulation 2007; 115:782-91.

25. Magne J, Sénéchal M, Mathieu P, et al. Restrictive annuloplasty for ischemic mitral regurgitation may induce functional mitral stenosis. J Am Coll Cardiol 2008;51:1692-701.

26. Agricola E, Oppizzi M, Pisani M, et al. Ischemic mitral regurgitation: mechanisms and echocardiographic classification. Eur J Echocardiogr 2008;9:207-21.

27. Hvass U, Tapia M, Baron F, et al. Papillary muscle sling: a new functional approach to mitral repair in patients with ischemic left ventricular dysfunction and functional mitral regurgitation. Ann Thorac Surg 2003;75:809-11.

28. Messas E, Guerrero JL, Handschumacher MD, et al. Chordal cutting: a new therapeutic approach for ischemic mitral regurgitation. Circulation 2001;104:1958-63.

29. De Bonis M, Lapenna E, La Canna G, et al. Mitral valve repair for functional mitral regurgitation in end-stage dilated cardiomyopathy: role of the "edge-to-edge" technique. Circulation 2005;112:1402-8.

30. Livi U, Alfieri O, Vitali E, et al. One-year clinical experience with the Acorn CorCap cardiac support device: results of a limited market release safety study in Italy and Sweden. Ital Heart J 2005;6:659-65

31. Vassiliades TA, Block PC, Cohn LH, et al. Society of Thoracic Surgeons; American Association for Thoracic Surgery; Society for Cardiovascular Angiography and Interventions. The clinical development of percutaneous heart valve technology. J Thorac Cardiovasc Surg 2005;129:970-6.

32. Kaye DM, Byrne M, Alferness C, et al. Feasibility and short-term efficacy of percutaneous mitral annular reduction for the therapy of heart failure-induced mitral regurgitation. Circulation 2003; 108:1795-7.

33. Dubreuil 0, Basmadjian A, Ducharme A, et al. Percutaneous mitral valve annuloplasty for ischemic mitral regurgitation: first in man experience with a temporary implant. Catheter Cardiovasc Interv 2007;69:1053-61.

34. Messas E, Bel A, Morichetti M, et al. Autologous myoblast transplantation for chronic ischemic mitral regurgitation. J Am Coll Cardiol 2006;47:2086-93. 